\title{
SUR LA SOMMATION DES SÉRIES DE LAPLACE ET DE LEGENDRE.
}

\author{
Par M. Michel Plancherel (Fribourg).
}

Adunanza del 13 agosto rgri.

Il existe des séries de polynômes de Legendre et des séries de fonctions sphériques divergeant en certains points bien que leurs fonctions génératrices soient continues. De telles séries peuvent être construites par les procédés généraux de MM. HAAR ${ }^{\text {I) }}$ et LebesGue ${ }^{2}$ ). Mais alors que pour une fonction sommable la convergence de la série de Fourier de cette fonction en un point ne dépend que des valeurs de la fonction au voisinage de ce point, il n'en est plus de même pour la série de Legendre de cette fonction. Témoin la fonction

$$
\frac{\mathrm{I}}{(\mathrm{I}-x)^{5}}, \quad-\mathrm{I} \leqslant x \leqslant \mathrm{I}
$$

dont la série de Legendre diverge dans tout l'intervalle $(-\mathrm{I},+\mathrm{I})$ lorsque $\left.\frac{3}{4} \leq s<\mathrm{I}^{3}\right)$. D'autre part, cependant, la suite des coefficients du développement en série de LaPLACE d'une fonction sommable détermine cette fonction d̀ une fonction d'intégrale nulle près. Il est donc important de connaitre des procédés simples permettant de reconstruire la fonction à partir des coefficients de son développement en série de Laplace. Le premier résultat général obtenu dans cette voie est dù à M. FéJeR ${ }^{4}$ ): la série de LAPLACE d'une fonction intégrable, sommée par les moyennes de Ceșíro du second ordre, converge vers la fonction en tout point de continuité de celle-ci.

Il y a quelques années, M. DE LA VALLée Poussin ${ }^{5}$ ) a été amené à considérer

I) A. HAAR, Zur Theorie der orthogonalen Funktionensysteme (I. Mitteilung) [Mathematische Annalen, Bd. LXIX (I9Io), pp. 33 I-371].

2) H. Lebesgue, Sur les intégrales singulières [Annales de la Faculté des Sciences de l'Université de Toulouse, III série, t. I (I909), pp. 25-II7].

3) L. Fejér, Über die Laplacesche Reibe [Mathematische Annalen, Bd. LXVII (1909), pp. 76-109].

A. HaAR, Über die Legendresche Reihe [Rendiconti del Circolo Matematico di Palermo, t. XXXII ( $2^{\mathrm{e}}$ sem. I9II), pp. I32-142].

4) loc. cit. ${ }^{3}$ ).

5) Ch.-J. de LA VAllée Poussin, Sur l'approximation des fonctions d'une variable réelle et de leurs dérivees par des polynómes et des suites limities de Fourier. [Bulletins de la classe des Sciences de l'Académie Royale de Belgique, 1908, pp. 193-254].

Rend. Circ. Matem. Palermo, t. XXXIII (1 ${ }^{\circ}$ sem. 1912). - Stampato il 3 gennajo 1912. 
un procédé de sommation entièrement différent des procédés de Cesàro et de Hölder. L'application de ce procédé aux séries de Fourier lui a donné des résultats d'une grande généralité. Son procédé de sommation lui permet non seulement de trouver la valeur de la fonction en tout poiut de continuité de celle-ci, mais encore de calculer les dérivées. de tout ordre de la fonction, là où elles existent.

Il m'a paru intéressant d'appliquer aux séries de LAPLACE un procédé de sommation du même type que celui de M. DE la Vallée Poussin. Les résultats obtenus ne le cèdent pas en généralité à ceux qu'il a obtenus sur les séries de Fourier; ils sont plus étendus que ceux que l'on obtient par l'emploi des secondes moyennes de Cesíro. Le procédé étudié ici présente encore sur celui de M. FÉJER l'avantage d'exprimer en formules simples et faciles à étudier les suites de fonctions dont la limite est cherchée ${ }^{6}$ ).

\section{ChAPITRE I.}

\section{La sommation de la série de Laplace.}

I. Le procédé de sommation. - Soit la série de fonctions

$$
u_{0}+u_{1}+\cdots+u_{n}+\cdots
$$

Le procédé de sommation de cette série, qu'emploie M. DE la Vallée Poussin consiste a poser

$$
\Sigma_{n}=u_{0}+\sum_{k=1}^{n} \frac{n(n-\mathrm{I}) \ldots(n-k+\mathrm{I})}{(n+\mathrm{I})(n+2) \ldots(n+k)} u_{k}
$$

et $\mathbf{d}$ chercher la limite de $\Sigma_{n}$ pour $n=\infty$. Si cette limite existe,

$$
\lim _{n=\infty} \Sigma_{n}=\mathbf{\Sigma}
$$

nous dirons que la série (I) est sommable par le procédé de M. DE LA VAllée Poussin et qu'elle a pour somme $\Sigma$. Nous n'emploierons pas ici le procidé (2), mais nous poserons

$$
S_{n}=u_{\mathrm{o}}+\sum_{k=1}^{n} \frac{n(n-\mathrm{I}) \ldots(n-k+\mathrm{I})}{(n+2)(n+3) \ldots(n+k+\mathrm{I})} u_{k}
$$

et nous chercherons la limite de $S_{n}$ pour $n=\infty$. Si cette limite existe,

$$
\lim _{n=\infty} S_{n}=S \text {, }
$$

nous dirons que la série (I) est sommable par le procédé $\left(S_{n}\right)$ et que sa somme est égale à $S$. Il est clair que $S$ peut exister sans que la série (I) soit convergente au sens ordinaire du mot, mais on voit sans difficulté que si la série (I) converge vers une limite $s$, la suite (3) converge aussi et que $S=s$. Pour le voir partons du lemme suivant:

6) Les résultats du présent travail ont été annoncés dans ma Note: Sur l'application aux séries de LAPLAce du procédé de sommation de M. DE LA VAltée Poussin [Comptes Rendus hehdomadaires des séances de l'Académie des Sciences (Paris), t. CLII (1 ${ }^{\text {er }}$ semestre I9II), pp. I226-1228]. 
Lemme : $a_{k}, b_{k}(k=\mathrm{I}, 2, \ldots, n)$ étant des quantités réelles, les $b_{k}$ étant décroissants et positifs, on a

si, quelque soit $k \leq n$,

$$
\left|\sum_{k=1}^{n} a_{k} b_{k}\right| \leq M b_{0}
$$

$$
\left|\sum_{p=1}^{k} a_{p}\right| \leq M
$$

Ce lemme résulte immédiatement de l'identité d'ABEL

$$
\sum_{k=1}^{n-} a_{k} b_{k}=\sum_{k=1}^{n=1}\left(b_{k}-b_{k+1}\right) \sum_{p=1}^{k} a_{p}+b_{n} \sum_{p=1}^{n} a_{p} .
$$

Supposant la série (I) convergente, nous pouvons, à tout $\varepsilon>0$ taire correspondre un indice $N=N(\varepsilon)$, tel que

puis, comme

$$
\left|\sum_{n}^{m} u_{k}\right|<\varepsilon, \text { pour } n, m \geqslant N
$$

$$
\lim _{n=\infty} \frac{n(n-\mathrm{I}) \ldots(n-k+\mathrm{I})}{(n+2)(n+3) \cdots(n+k+\mathrm{I})}=\mathbf{I}
$$

lorsque $k$ reste fixe, nous déterminerons $M=M(\mathrm{~s})$, tel que

$$
\left|\sum_{k=1}^{N} \frac{m(m-\mathrm{I}) \ldots(m-k+\mathrm{I})}{(m+2) \ldots(m+k+\mathrm{I})} u_{k}-\sum_{k=\mathrm{I}}^{N} u_{k}\right|<\varepsilon, \text { pour } m \supseteq M .
$$

Or, comme pour toute valeur de $m$ supérieure au plus grand des deux nombres $M, N$

$$
S_{m}=\sum_{k=1}^{N} \frac{m(m-\mathrm{I}) \ldots(m-k+\mathrm{I})}{(m+2) \ldots(m+k+\mathrm{I})} u_{k}+\sum_{k=N^{+}+1}^{m} \frac{m(m-1) \ldots(m-k+\mathrm{I})}{(m+2) \ldots(m+k+\mathrm{I})} u_{k}
$$

et que le lemme appliqué à la seconde somme du second membre montre qu'elle est plus petite en valeur absolue que

on conclut

$$
\varepsilon \cdot \frac{m(m-1) \ldots(m-N)}{(m+\mathrm{I}) \ldots(m+N+2)}<\varepsilon
$$

c'est-à-dire

$$
\left|S_{m}-\sum_{k=1}^{N} u_{k}\right|<2 \varepsilon, \quad m \geqslant M, N
$$

$$
\lim _{n=\infty} S_{n}=\lim _{N=\infty} \sum_{1}^{N} u_{k}=s .
$$

Le procédé de sommation $\left(S_{n}\right)$ est du même type que le procédé $\left(\Sigma_{n}\right)$ de M. DE La Vallée Poussin. Il se pose naturellement à son sujet la question de savoir s'il lui est équivalent. La réponse est affirmative, elle s'exprime par le théorème suivant:

Si la série (I) est sommable par le procédé $\left(\mathbf{\Sigma}_{n}\right)$, elle est sommable, de même somme, par le procédé $\left(S_{n}\right)$, et réciproquement.

Je donne de ce théorème une démonstration que je dois à l'obligeance de mon 
ami M. Hermann Weyl. Exprimons $S_{n}$ linéairement en fonction de $\mathbf{\Sigma}_{0}, \mathbf{\Sigma}_{1}, \ldots, \mathbf{\Sigma}_{n}$

$$
\begin{aligned}
& S_{0}=\alpha_{00} \Sigma_{0}, \\
& S_{\mathrm{r}}=\alpha_{\mathrm{so}} \Sigma_{\mathrm{o}}+\alpha_{11} \Sigma_{1}, \ldots \ldots \ldots \ldots . \\
& \cdots \cdots \alpha_{n n} \Sigma_{n} .
\end{aligned}
$$

C'est évidemment possible. Les coefficients $\alpha_{n, k}$ sont indépendants de $u_{o}, u_{1}, \ldots, u_{n}$; ils ne dépendent que de $n$ et $k$. Leur calcul, au moyen de (2) et (3), est assez long, mais simple. On trouve

$$
\begin{aligned}
& \alpha_{n o}=\frac{\mathrm{I}}{2 n+\mathrm{I}}, \\
& \alpha_{n}=\frac{\mathrm{I}}{\mathrm{I}} \cdot \frac{n+\mathrm{I}}{(2 n+\mathrm{I})(2 n-\mathrm{I})}, \\
& \alpha_{n k}=\frac{\mathrm{I} \cdot 3 \cdot 5 \ldots(2 k-\mathrm{I})}{k !} \frac{(n+\mathrm{I}) n(n-\mathrm{I}) \ldots(n-k+2)}{(2 n+\mathrm{I})(2 n-\mathrm{I}) \cdots(2 n-2 k+\mathrm{I})} \quad(k \leq n), \\
& \alpha_{n, n}=\frac{n+\mathrm{I}}{2 n+\mathrm{I}} \text {. }
\end{aligned}
$$

En égalant dans l'expression de $S_{n}$ les coefficients de $u_{0}$ dans les deux membres, il vient

$$
\alpha_{n o}+\alpha_{n 1}+\cdots+\alpha_{n n}=\mathrm{r} .
$$

Les formules trouvées montrent encore que, pour $k, N$ constants quelconques,

$$
\begin{gathered}
\lim _{n=\infty} \alpha_{n k}=0, \\
\lim _{n=\infty} \sum_{k=0}^{N} \alpha_{n k}=0, \quad \lim _{n=\infty} \sum_{k=N+1}^{n} \alpha_{n k}=\mathrm{I} .
\end{gathered}
$$

Si done

$$
\lim _{n=\infty} \mathbf{\Sigma}_{n}=\mathbf{\Sigma}
$$

nous pourrons, à tout $\varepsilon>0$, faire correspondre un entier $N=N(\Sigma)$ par la condition

Décomposant alors

$$
\left|\Sigma_{n}-\Sigma\right|<\varepsilon, \quad n \gg N
$$

$$
\begin{aligned}
S_{n} & =\sum_{k=0}^{N} \alpha_{n k} \Sigma_{k}+\sum_{k=N+\mathrm{r}}^{n} \alpha_{n k} \Sigma_{k} \\
& =\mathbf{\Sigma} \sum_{k=N^{+} \mathrm{r}}^{n} \alpha_{n k}+\sum_{k=0}^{N} \alpha_{n k} \Sigma_{k}+\sum_{k=N+\mathrm{r}}^{n-} \alpha_{n k}\left(\Sigma_{k}-\Sigma\right)
\end{aligned}
$$

on voit que la première somme du second membre tend vers $\mathbf{\Sigma}$ lorsque $n$ tend vers l'infini; la seconde tend dans les mêmes conditions vers zéro; quant à la troisième, elle est en valeur absolue plus petite que $\varepsilon$, puisque tous les $x_{n k}$ sont positifs et de somme au plus égale d̀ $\mathrm{I}$. On a donc, puisque $\varepsilon$ est arbitrairement petit,

$$
\lim _{n=\infty} S_{n}=\Sigma \text {. }
$$


Inversement, si l'on exprime $\mathbf{s}_{n}$ linéairement en fonction de $S_{0}, S_{1}, \ldots, S_{n}$

$$
\begin{aligned}
& \Sigma_{0}=\beta_{00} s_{0}, \\
& \Sigma_{1}=\beta_{10} s_{0}+\beta_{11} s_{1}, \\
& \Sigma_{n}=\beta_{n 0} s_{0}+\beta_{n 1} s_{1}+\cdots+\beta_{n n} s_{n},
\end{aligned}
$$

les coefficients $\beta_{n k}$ sont donnés par

$$
\begin{aligned}
& \beta_{n o}=-\frac{1}{2(2 n-\mathrm{I})}, \\
& \beta_{n 1}=-\frac{1 \cdot 3}{2.2 !} \frac{n}{(2 n-\mathrm{I})(2 n-3)} \text {, } \\
& \beta_{n k}=-\frac{1.3 .5 \ldots(2 k+\mathrm{I})}{2(k+\mathrm{I}) !} \frac{n(n-\mathrm{I}) \ldots(n-k+\mathrm{I})}{(2 n-\mathrm{I})(2 n-3) \ldots(2 n-2 k-\mathrm{I})}, \\
& \beta_{n, n-1}=-\frac{1}{2} \text {, } \\
& \beta_{n, n}=\frac{2 n+\mathrm{I}}{n+\mathrm{I}}
\end{aligned}
$$

Ici encore

$$
\begin{aligned}
\beta_{n 0}+\beta_{n \mathrm{I}}+\cdots+\beta_{n n} & =\mathrm{I}, \\
\lim _{n=\infty} \beta_{n k} & =0 .
\end{aligned}
$$

Tous les coefficients $\beta_{n k}$ sont négatifs, sauf $\beta_{n n}$. On peut donc démontrer de la même manière que si

existe, l'on a aussi

$$
\lim _{n=\infty} S_{n}=S
$$

$$
\lim _{n=\infty} \Sigma_{n}=S \text {. }
$$

Il y a donc bien équivalence complète entre les deux procédés de sommation. Tous les théorèmes des $\$ suivants sont donc encore vrais, si l'on remplace dans leur énoncé le procéde $\left(S_{n}\right)$ par le procédé $\left(\Sigma_{n}\right)$ de M. DE La Vallée Poussin, car ces théorèmes ne concernent que la limite de $S_{n}$. Les formules se présentant, dans ce qui suit, beaucoup plus simplement lorsqu'on emploie le procédé $\left(S_{n}\right)$, nous nous bornerons à l'emploi de ce dernier.

2. Quelques formules préliminaires, - Partons de la relation

$$
\left(2 \cos \frac{x}{2}\right)^{2 n+1}=\left(e^{\frac{i x}{2}}+e^{-\frac{i x}{2}}\right)^{2 n+1},
$$

développons le second membre par la formule du binôme et groupons deux à deux les termes du développement également éloignés des extrêmes. Nous obtenons

$$
\begin{aligned}
2\left[\cos \left(n+\frac{\mathrm{I}}{2}\right)\right. & x+\left(\begin{array}{c}
2 n+\mathrm{I} \\
\mathrm{I}
\end{array}\right) \cos \left(n-\frac{\mathrm{I}}{2}\right) x+\cdots \\
\cdots & \left.+\left(\begin{array}{c}
2 n+\mathrm{I} \\
k
\end{array}\right) \cos \left(n-k+\frac{\mathrm{I}}{2}\right) x+\cdots+\left(\begin{array}{c}
2 n+\mathrm{I} \\
n
\end{array}\right) \cos \frac{x}{2}\right] .
\end{aligned}
$$


Renversons l'ordre des termes et mettons $\left(\begin{array}{c}2 n+1 \\ n\end{array}\right)$ en facteur:

$$
2^{2 n+1} \cos ^{2 n+1} \frac{x}{2}=2\left(\begin{array}{c}
2 n+\mathrm{I} \\
n
\end{array}\right)\left[\cos \frac{x}{2}+\sum_{k=1}^{n} \frac{n(n-\mathrm{I}) \ldots(n-k+\mathrm{I})}{(n+2)(n+3) \ldots(n+k+\mathrm{I})} \cos \left(k+\frac{\mathrm{I}}{2}\right) x\right] .
$$

Dérivons par rapport à $x$; nous obtenons, après quelques réductions faciles,

(4) $\left\{\begin{array}{c}\frac{2^{2 n}(n !)^{2}(n+\mathrm{I})}{(2 n) !} \cos ^{2 n} \frac{x}{2} \sin \frac{x}{2} \\ =\sin -\frac{x}{2}+\sum_{k=1}^{n} \frac{n(n-\mathrm{I}) \ldots(n-k+\mathrm{I})}{(n+2)(n+3) \ldots(n+k+\mathrm{I})}(2 k+\mathrm{I}) \sin \left(k+\frac{\mathrm{I}}{2}\right) x .\end{array}\right.$

Cette identité est très importante. Elle nous permettra de trouver une expression simple de la sommation des séries de Laplace. Désignons par $k_{n}$ la quantité

$$
k_{n}=\frac{2^{2 n} \cdot(n !)^{2}(n+1)}{\pi(2 n) !}
$$

et cherchons-en une expression asymptotique pour $n$ très grand. On peut utiliser pour celd la formule de Striring, qui donne

$$
n !=\sqrt{2 n} n^{n} e^{n}\left(\sqrt{\pi}+\frac{\omega}{\sqrt{2 n}}\right)
$$

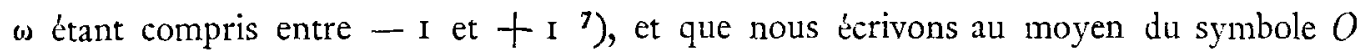
de M. Landau:

$$
n !=\sqrt{2 n} \cdot n^{n} e^{n}\left(1^{-\pi}+O\left(n^{-\frac{1}{2}}\right)\right)
$$

Ce symbole a ici la signification suivante: $g(n)$ étant une fonction de l'argument discontinu $n$, positive pour les grandes valeurs de $n$, et $f(n)$ étant une fonction quelconque de $n$, l'écriture

$$
f(n)=O(g(n))
$$

veut dire qu'à partir d'une certaine valeur $N$ de $n$

$$
\left|\frac{f(n)}{g(n)}\right|<A, \quad n \gg N
$$

$A$ étant une constante positive indépendante de $n$. Lorsque $f(n)$ contient encore un paramètre variable $t$, et que, dans un certain intervalle $x \leqslant t \leqslant \beta$,

$$
\left|\frac{f(n)}{g(n)}\right|<A, \quad n \geqq N, \quad \alpha \leq t \leq \beta,
$$

$A$ étant indépendant de $n, t$, nous dirons que $f(n)=O(g(n))$ uniformément en $t$ dans $(\alpha, \beta)$. On voit au moyen de cette notation que

$$
k_{n}=\frac{(n+\mathrm{I}) \sqrt{n \pi}}{\pi}+O(n)=O\left(n^{\frac{3}{2}}\right) .
$$

7) Voir, par ex : É. Goursat, Cours d'Analyse (Paris, Gauthier-Villars), tome I (1902), page 328 ; C. Jordan, Cours d'Analyse, $2^{\mathrm{e}}$ édition (Paris, Gauthier-Villars), tome II (I894), page 182. 
$P_{n}(x)$ désignant comme d'habitude le polynôme de Legendre d'ordre $n$,

$$
P_{n}(x)=\frac{\mathrm{I}}{2^{n} n !} \frac{d^{n}\left(x^{2}-\mathrm{I}\right)^{n}}{d x^{n}},
$$

nous aurons à utiliser la formule de Mehler ${ }^{8}$ )

$$
P_{n}(\cos \omega)=\frac{2}{\pi} \int_{\omega}^{\pi} \frac{\sin \left(n+\frac{1}{2}\right) \psi d \psi}{\sqrt{2(\cos \omega-\cos \psi)}} \quad(n=0,1,2, \ldots ; 0 \leqslant \omega \leqslant \pi)
$$

qui, pour $n=\mathrm{o}$, se réduit à

$$
I=\frac{2}{\pi} \int_{\omega}^{\pi} \frac{\sin \frac{1}{2} \psi d \psi}{\sqrt{2(\cos \omega-\cos \psi)}} .
$$

Le radical est $\grave{a}$ prendre positivement dans ces formules.

3. La série de Laplace. - Sur la surface sphérique de rayon égal à l'unité

$$
x^{2}+y^{2}+z^{2}=\mathrm{I}
$$

introduisons les coordonnées polaires par les formules

$$
\begin{gathered}
x=\sin \ni \cos \varphi, \quad y=\sin \ni \sin \varphi, \quad z=\cos \ni \\
0 \leqslant \Im \leqslant \pi, \quad 0 \leqslant \varphi \leqslant 2 \pi .
\end{gathered}
$$

L'axe des $z$ est donc pris comme axe polaire, $\ni$ est la distance (sphérique) polaire et $\varphi$ la longitude. $(\ni, \varphi)$ et $\left(\nexists^{\prime}, \varphi^{\prime}\right)$ étant deux points de la surface sphérique, leur distance sphérique $\omega$ est donnée par

$$
\cos \omega=\cos \ni \cos \jmath^{\prime}+\sin \ni \sin \jmath^{\prime} \cos \left(\varphi-\varphi^{\prime}\right) .
$$

L'elément d'aire au point $\left(J^{\prime}, \varphi^{\prime}\right)$ a pour valeur

$$
d \sigma^{\prime}=\sin s^{\prime} d \Xi^{\prime} d \varphi^{\prime} \text {. }
$$

La formule suivante de la théorie des fonctions sphériques

$$
\int P_{n}(\cos \omega) d \sigma^{\prime}=\mathrm{o}
$$

où l'intégrale (comme ce sera toujours le cas, sans indication expresse du contraire) est étendue à toute la surface sphérique, est une conséquence inımédiate du fait que sa valeur est indépendante de la position du point $(z, \varphi)$ et de la propriété d'orthogonalité des polynômes de LEGENDRE

$$
\begin{aligned}
\int P_{n}(\cos \omega) d \sigma^{\prime}=\int P_{n}\left(\cos \Im^{\prime}\right) d \sigma^{\prime} & =\int_{0}^{\pi} d \Im^{\prime} \sin \Im^{\prime} P_{n}\left(\cos \Im^{\prime}\right) \int_{0}^{2 \pi} d \rho^{\prime} \\
& =2 \pi \int_{-1}^{+\pi} P_{n}(x) d x=0 \quad(n>0) .
\end{aligned}
$$

Considérons maintenant une fonction $F(\jmath, \varphi)$ de la position d'un point sur la

8) Cfr. Herne, Handbuch der Kugelfunctionen (Berlin, G. Reimer), Bd. I, 2. Auflage (1878), p. 44. Cette formule résulte très simplement des formules de DiRichLET. 
sphère et faisons la seule hypothèse que l'intégrale de la valeur absolue de cette fonction, étendue à toute la surface sphérique, existe:

$$
\int\left|F\left(\Im^{\prime}, \varphi^{\prime}\right)\right| d \sigma^{\prime}=M
$$

Cette intégrale peut s'entendre au sens ordinaire de Riemann ou au sens de LebesGue. Nos raisonnements sont les mêmes dans un cas et dans l'autre. Nous pouvons sous cette hypothèse former effectivement tous les termes de la série suivante, convergente ou non,

$$
\frac{\mathrm{I}}{4 \pi} \int F\left(\Im^{\prime}, \varphi^{\prime}\right) d \sigma^{\prime}+\sum_{k=1}^{\infty} \frac{2 k+\mathrm{I}}{4 \pi} \int F\left(\Im^{\prime}, \varphi^{\prime}\right) P_{k}(\cos \omega) d \sigma^{\prime} .
$$

C'est la série de Laplace de $F(\approx, \varphi)$. Sommant cette série par le procédé indiqué plus haut (3), nous avons à former la suite

$$
\text { (I3) }\left\{\begin{array}{c}
S_{n}(\jmath, \varphi)=\frac{\mathrm{I}}{4 \pi} \int F\left(\tau^{\prime}, \varphi^{\prime}\right) d \sigma^{\prime} \\
+\sum_{k=1}^{n} \frac{2 k+\mathrm{I}}{4 \pi} \frac{n(n-\mathrm{I}) \ldots(n-k+\mathrm{I})}{(n+2)(n+3) \cdots(n+k+\mathrm{I})} \int F\left(\Im^{\prime}, \varphi^{\prime}\right) P_{k}(\cos \omega) d \sigma^{\prime}
\end{array}\right.
$$

et à chercher sa limite pour $n=\infty$. Appliquant la formule de Mehler (7) et l'identité (4), nous obtenons

$$
S_{n}(\tau, \varphi)=\frac{k_{n}}{2 \pi} \int d \sigma^{\prime} F\left(J^{\prime}, \varphi^{\prime}\right) \int_{\omega}^{\pi} \cos ^{2 n} \frac{\psi}{2} \frac{\sin \frac{\psi}{2} d \psi}{\sqrt{2(\cos \omega-\cos \psi)}} .
$$

L'expression asymptotique (6) de $k_{n}$ montre qu'il est équivalent de chercher la limite pour $n=\infty$ de $S_{n}(\neg, q)$ ou de

$$
\frac{(n+\mathrm{I}) 1^{\prime} n \pi}{2 \pi^{2}} \int d \sigma^{\prime} F\left(\Xi^{\prime}, \psi^{\prime}\right) \int_{\omega}^{\pi} \cos ^{2 n} \frac{\psi}{2} \frac{\sin \frac{\psi}{2} d \psi}{\sqrt{2(\cos \omega-\cos \psi)}} .
$$

Notons, pour abréger,

donc

$$
L(n, t)=\int_{t}^{\pi} \cos ^{2 n} \frac{\psi}{2} \frac{\sin \frac{\psi}{2} d \psi}{\sqrt{2(\cos t-\cos \psi)}} \quad(\circ \leqslant t \leqslant \pi),
$$

$$
S_{n}(\jmath, \varphi)=\frac{k_{n}}{2 \pi} \int F\left(\sigma^{\prime}, \varphi^{\prime}\right) L(n, \omega) d \sigma^{\prime}
$$

4. Propriétés de $L(n, t)$. - Il résulte de sa définition (IS) que $L(n, t)$ est une quantité essentiellement positive à l'intérieur de l'intervalle $(0, \pi)$,

$$
L(n, t)>0 \quad(0<t<\pi) .
$$

Cette remarque est importante; elle nous permettra plus loin de nous servir uniquement du premier théorème de la moyenne dans les estimations d'intégrales que nous ferons. 
Si nous prenons maintenant pour $F(\nexists, \varphi)$ la fonction

$$
F(\tau, \varphi)=\mathrm{I} \text {, }
$$

les formules (IO) montrent que la série de LAPLACE (I2) se réduit à son premier terme; on a donc ici

d'où, d'après (I6),

$$
S_{n}(\nabla, \varphi)=\mathrm{I}
$$

$$
\frac{k_{n}}{2 \pi} \int L(n, \omega) d \sigma^{\prime}=\mathrm{I} \text {. }
$$

$\cos ^{2 n} \frac{t}{2}$ étant une fonction positive décroissante dans $(0, \pi)$, le premier théorème de la moyenne donne dans (IS)

c'est-d̀-dire, d'après $\left(7_{a}\right)$,

$$
L(n, t)<\cos ^{2 n} \frac{t}{2} \int_{t}^{\pi} \frac{\sin \frac{\psi}{2} d \psi}{\sqrt{2(\cos t-\cos \psi)}},
$$

Or, on a

$$
L(n, t)<\frac{\pi}{2} \cos ^{2 n} \frac{t}{2}=O\left(\cos ^{2 n} \frac{t}{2}\right) .
$$

Mais,

$$
\begin{aligned}
\cos \frac{t}{2} & =\mathrm{I}-\frac{\mathrm{I}}{2 !}\left(\frac{t}{2}\right)^{2}+\frac{\mathrm{I}}{4 !}\left(\frac{t}{2}\right)^{4}-\ldots \\
& <\mathrm{I}-\frac{\mathrm{I}}{2 !}\left(\frac{t}{2}\right)^{2}+\frac{\mathrm{I}}{4^{2}}\left(\frac{t}{2}\right)^{4}=\left(\mathrm{I}-\frac{t^{2}}{\mathrm{I} 6}\right)^{2} .
\end{aligned}
$$

donc,

$$
\mathrm{I}-\frac{t^{2}}{\mathrm{I} 6}<e^{-\frac{t^{2}}{16}}
$$

c'est-à-dire

$$
\begin{aligned}
\cos \frac{t}{2} & <e^{-\frac{t^{2}}{8}}, \\
\cos ^{2 n} \frac{t}{2} & <e^{-\frac{n t^{2}}{4}},
\end{aligned}
$$

Donc,

$$
\cos ^{2 n} \frac{t}{2}=O\left(e^{-n \frac{t^{2}}{4}}\right)
$$

et il résulte des inégalités trouvées que, dans tout intervalle $\varepsilon \leqslant t \leqslant \pi, \varepsilon>0$, on a uniformément en $t$

$$
\frac{k_{n}}{2 \pi} L(n, t)=O\left(n^{\frac{3}{2}} e^{-n \frac{t^{2}}{4}}\right)
$$

Si donc on étend l'intégrale suivante à la calotte déterminée par l'inégalité $\omega \gg \varepsilon$, il vient

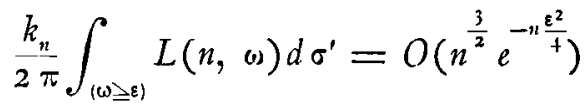


ce qui montre que cette intégrale tend vers zéro avec $\frac{\mathrm{I}}{n}$. Il vient donc, à cause de (I8),

$$
\frac{k_{n}}{2 \pi} \int_{(\omega \leq \varepsilon)} L(n, \omega) d \sigma^{\prime}=\mathrm{I}-O\left(n^{\frac{3}{2}} e^{-n \frac{\varepsilon^{2}}{4}}\right)
$$

l'intégrale étant étendue, comme le montre la notation, à l'aire de la calotte de centre $(\exists$, p) et de rayon sphérique $\varepsilon$. Par suite

$$
\lim _{n=\infty} \frac{k_{n}}{2 \pi} \int_{(\omega \leq \varepsilon)} L(n, \omega) d \sigma^{\prime}=\mathrm{I} .
$$

5. La convergence de la suite $S_{n}(\Im, \varphi)$. - Les estimations précédentes permettent de démontrer rapidement la convergence de la suite $S_{n}(\tau, \varphi)$ sous des conditions déjà très générales. Énonçons d'abord le

ThÉorème I. - La convergence de la suite $S_{n}(\tau, \varphi)$ en un point ne dépend que des valeurs de la fonction $F(z, \varphi)$ au voisinage de ce point.

Par voisinage du point $(\Im, p)$ nous entendrons toujours l'ensemble des points vérifiant la condition

$$
0<\omega \leqslant \varepsilon,
$$

$\varepsilon$ étant une quantité positive. Ce voisinage est donc formé par l'ensemble de tous les points de la calotte $\omega \leqslant \varepsilon$, à l'exception du point $(\tau, \varphi)$ lui-même. D'́composons maintenant la sphère en deux calottes par le petit cercle $\omega=\varepsilon$; nous avons, en tenant compte des estimations du $\ 4$,

$$
\begin{aligned}
\left|\frac{k_{n}}{2 \pi} \int_{(\omega \supseteq \varepsilon)} F\left(\Im^{\prime}, \varphi^{\prime}\right) L(n, \omega) d \sigma^{\prime}\right| & \leq \frac{k_{n}}{2 \pi} \max _{\varepsilon \leq \omega \leq \pi} L(n, \omega) \cdot \int_{(\omega \supseteq \varepsilon)}\left|F\left(\jmath^{\prime}, \varphi^{\prime}\right)\right| d \tau^{\prime} \\
& =O\left(n^{\frac{3}{2}} e^{-n \frac{\varepsilon^{2}}{4}}\right) .
\end{aligned}
$$

On a donc, pour tout $\varepsilon>0$,

$$
\lim _{n=\infty} S_{n}(\tau, \varphi)=\lim _{n=\infty} \frac{k_{u}}{2 \pi} \int_{(\omega \leq \varepsilon)} F\left(\jmath^{\prime}, \varphi^{\prime}\right) L(n, \omega) d \sigma^{\prime},
$$

ce qui démontre le théorème.

Si nous supposons maintenant que la fonction $F\left(J^{\prime}, q^{\prime}\right)$ est bornée dans le voisinage $o<\omega \leqslant \varepsilon$ et $\operatorname{si} m(\varepsilon)$ et $M(\varepsilon)$ sont ses limites inférieure et supérieure dans ce voisinage, nous voyons, en appliquant à (23) le théorème de la moyenne, que $m(\varepsilon) \frac{k_{n}}{2 \pi} \int_{(\omega \leq \varepsilon)} L(n, \omega) d \sigma^{\prime} \leq \frac{k_{n}}{2 \pi} \int_{(\omega \leq \varepsilon)} F\left(\tau^{\prime}, \varphi^{\prime}\right) L(n, \omega) d \sigma^{\prime} \leq M(\varepsilon) \frac{k_{n}}{2 \pi} \int_{(\omega \leq \varepsilon)} L(n, \omega) d \sigma^{\prime} ;$ donc, (22), en passant à la limite,

$$
m(\varepsilon) \leq \lim _{n=\infty} \text { inf. } S_{n}(\tau, \rho) \leq \lim _{n=\infty} \text { sup. } S_{n}(\approx, \psi) \leq M(\varepsilon)
$$

ce que nous pouvons énoncer:

THÉORÈme II. - Les limites inférieure et supérieure pour $n=\infty$ de la suite $S_{n}(\pi, j)$ en un point, sont comprises entre les limites inférieure et supérieure de $F(\mathrm{~J}, p)$ dans le voisinage de ce point. 
$\mathrm{Si}$, en particulier, la fonction $F$ est bornée sur toute la sphère, on voit, en reprenant l'analyse, que:

Les sommes $S_{n}(\nexists, \varphi)$ relatives à une fonction $F(\pi, \varphi)$ bornée sur toute la surface sphérique, restent comprises entre les limites inférieure et supérieure de F sur la surface.

Si maintenant $F$ est continue au point $(\exists, p)$,

$$
\lim _{\varepsilon=0} m(\varepsilon)=\lim _{\varepsilon=0} M(\varepsilon)=F(\tau, \varphi) \text {, }
$$

et le passage à la limite $\varepsilon=0$ dans (24) donne

$$
\lim _{n=\infty} S_{n}(J, \varphi)=F(j, \varphi) \text {. }
$$

Si $F(J, \varphi)$ est continue dans toute une calotte, on voit, en reprenant les raisonnements, que la convergence de $S_{n}(j, \psi)$ vers sa limite a lieu uniformément dans toute calotte entièrement intérieure à la première. Nous avons donc, en résumé, le

Théorème III. - La suite $S_{n}(\Im, \varphi)$ a pour limite $F(s, \uparrow)$ en tout point de continuité de la fonction $F$. La convergence est uniforme dans toute calotte entièrement intérieure à une calotte de continuite de $F(\tau, \varphi)$.

6. Introduction de la valeur mojenne. - Soit $(\Omega, \psi)$ un point de la surface sphérique. Formons l'intégrale de ligne

$$
F(\tau, \varphi ; t)=\frac{I}{s(t)} \int_{\omega=t} F\left(\tau^{\prime}, \varphi^{\prime}\right) d s
$$

étendue au petit cercle de centre $(\tau, \uparrow)$ et de rayon sphérique égal à $t$. $d s$ est l'élément d'arc sur ce petit cercle et $s(t)$ désigne son périmètre. La limite

$$
\lim _{t= \pm+0} F(\tau, \rho ; t)=F(\tau, \varphi ;+0)
$$

si elle existe, est dite la valeur mojenne de la fonction $F$ au point (J, $p$ ). Lorsque la fonction $F$ est continue au point $(\exists, \varphi)$, sa valeur moyenne en ce point existe et est égale d $F$; on le voit, sans difficulté, en remarquant que dans ce cas

$$
F\left(\nexists^{\prime}, \varphi^{\prime}\right)=F(\nexists, \varphi)+\lambda\left(\nexists^{\prime}, \varphi^{\prime}\right),
$$

$\lambda\left(\nexists^{\prime}, p^{\prime}\right)$ tendant uniformément vers zéro avec $\omega$. L'introduction de la valeur moyenne permet de démontrer le théorème suivant, dont la première partie du théorème III est un cas particulier.

THÉORÈme IV. - La suite $S_{n}(\tau, \varphi)$ a pour limite la valeur moyenne $F(\nexists, \varphi ;+0)$ en tout point où cette expression existe.

La généralité de la démonstration n'est pas diminuée en supposant, pour simplifier l'écriture, que le point $(\nexists, \varphi)$ est au pôle du système de coordonnées, puisque la valeur des termes de la série de Laplace est indépendante de la position de ce système. $F(\exists, \varphi ; t)$ se réduit alors à

$$
F(0, \varphi ; t)=f(t)=\frac{1}{2 \pi} \int_{0}^{2 \pi} F\left(t, \varphi^{\prime}\right) d \varphi^{\prime} .
$$


La formule (16) donne au pôle, car alors $s=0, \omega=\jmath^{\prime}$,

$$
\begin{aligned}
S_{n} & =\frac{k_{n}}{2 \pi} \int F\left(\nexists^{\prime}, \varphi^{\prime}\right) L\left(n, \Im^{\prime}\right) d \sigma^{\prime} \\
& =\frac{k_{n}}{2 \pi} \int_{0}^{\pi} d \Im^{\prime} \sin \Im^{\prime} L\left(n, \Im^{\prime}\right) \int_{0}^{\pi} F\left(\Im^{\prime}, \varphi^{\prime}\right) d \varphi^{\prime} \\
& =k_{n} \int_{0}^{\pi} f(t) L(n, t) \sin t d t .
\end{aligned}
$$

Les relations (18) et (22) donnent également

$$
k_{n} \int_{0}^{\pi} L(n, t) \sin t d t=\mathrm{I},
$$

$$
\lim _{n=\infty} k_{n} \int_{0}^{\varepsilon} L(n, t) \sin t d t=\mathrm{I}
$$

et il vient, d'après le théorème $I$,

$$
\lim _{n=\infty} S_{n}=\lim _{n=\infty} k_{n} \int_{0}^{\varepsilon} f(t) L(n, t) \sin t d t .
$$

Or, $L(n, t) \sin t$ étant positif, il vient, par l'emploi du théorème de la moyenne, $\bar{m}(\varepsilon)$ et $\vec{M}(\varepsilon)$ représentant ici les limites inférieure et supérieure de $f(t)$ dans o $<t \leq \varepsilon$, $\left(24_{a}\right)$

$$
\bar{m}(\varepsilon) \leq \lim _{n=\infty} \text { inf. } S_{n} \leq \lim _{n=\infty} \sup . S_{n} \leq \bar{M}(\varepsilon),
$$

c'est-ḋ-dire au cas où $f(+0)$ existe

$$
\begin{aligned}
\lim _{n=\infty} S_{n} & =\lim _{\varepsilon=0} \bar{m}(e)=\lim _{\varepsilon=0} \bar{M}(\varepsilon) \\
& =f(+0) .
\end{aligned}
$$

7. La dérivée première de $L(n, t)$. - Avant d'étudier des cas de convergence plus généraux et de passer à l'étude des dérivées de la suite $S_{n}(\tau, \varphi)$, il est nécessaire d'étudier les dérivées de $L(n, t)$. Supposons d'abord $t \supseteq \frac{\pi}{2}$. De (I $)$ résulte alors, en effectuant la transformation

et en posant

$$
\begin{aligned}
u & =-\cos \psi \\
d u & =2 \sin \frac{\psi}{2} \cos \frac{\psi}{2} d \psi
\end{aligned}
$$

la valeur

$$
z=-\cos t
$$

Donc,

$$
L(n, t)=\frac{\mathrm{I}}{2 \sqrt{2}} \int_{z}^{1} \cos ^{2 n-1} \frac{\psi}{2} \frac{d u}{\sqrt{u-z}} .
$$

$$
\begin{aligned}
\frac{d L(n, t)}{d t} & =\frac{d L(n, t)}{d z} \frac{d z}{d t} \\
& =\frac{1}{2 \sqrt{2}} \sin t \frac{d}{d z} \int_{z}^{t} \cos ^{2 n-1} \frac{\psi}{2} \frac{d u}{\sqrt{u-z}} .
\end{aligned}
$$


Par une intégration par parties

$$
\int_{z}^{1} \cos ^{2 n-1} \frac{\psi}{2} \frac{d u}{\sqrt{u-z}}=\left|2 \cos ^{2 n-1} \frac{\psi}{2} \cdot \sqrt{u-z}\right|_{u=z}^{u=1}-2 \int_{z}^{1} \frac{d}{d u} \cos ^{2 n-1} \frac{\psi}{2} \cdot \sqrt{u-z} d u .
$$

Le terme aux limites est nul, car $\cos \frac{\psi}{2}$ s'annule pour $u=\mathrm{I}$. Il vient maintenant par différentiation

$$
\begin{aligned}
\frac{d}{d z} \int_{z}^{1} \cos ^{2 n-1} \frac{\psi}{2} \frac{d u}{\sqrt{u-z}} & =-2 \frac{d}{d z} \int_{z}^{1} \frac{d}{d u} \cos ^{2 n-1} \frac{\psi}{2} \cdot \sqrt{u-z} d u \\
& =-\frac{2 n-1}{2} \int_{\tau}^{t} \cos ^{2 n-2} \frac{\psi}{2} \cdot \sin \frac{\psi}{2} \frac{d \psi}{d u} \cdot \frac{d u}{\sqrt{u-z}} \\
& =-\frac{2 n-1}{2} \int_{t}^{\pi} \cos ^{2 n-2} \frac{\psi}{2} \cdot \frac{\sin \frac{\psi}{2} d \psi}{\sqrt{\cos t-\cos \psi}} .
\end{aligned}
$$

Par suite,

$$
\frac{d L(n, t)}{d t}=-\frac{2 n-1}{4} \sin t \int_{t}^{\pi} \cos ^{2 n-2} \frac{\psi}{2} \frac{\sin \frac{\psi}{2} d \psi}{\sqrt{2(\cos t-\cos \psi)}}
$$

ou

$$
\frac{d L(n, t)}{d t}=-\frac{2 n-\mathrm{I}}{4} \sin t L(n-\mathrm{I}, t) .
$$

Cette formule, vraie pour $t \geqslant \frac{\pi}{2}$, s'etend par prolongement analytique d tout l'intervalle $(0, \pi)$, car $L(n, t)$ et $L(n-\mathrm{I}, t)$ sont des fonctions entières de $t$, comme il résulte de (I3). On peut d'ailleurs la démontrer directement pour $t<\frac{\pi}{2}$ en décomposant $L(n, t)$ en deux intégrales

$$
L_{1}(n, t)=\int_{t}^{\frac{\pi}{2}} \cos ^{2 n} \frac{\psi}{2} \frac{\sin \frac{\psi}{2} d \psi}{\sqrt{2(\cos t-\cos \psi)}}, \quad L_{2}(n, t)=\int_{\frac{\pi}{2}}^{\pi} \cos ^{2 n} \frac{\psi}{2} \frac{\sin \frac{\psi}{2} d \psi}{\sqrt{2(\cos t-\cos \psi)}} .
$$

La substitution

$$
\begin{aligned}
& \qquad \begin{aligned}
u & =\cos \psi, \\
z & =\cos t \\
u & =-\cos \psi, \\
\text { pour la première et } \quad z & =\cos t
\end{aligned} \\
& \text { pour la seconde, les ramènent à } \\
& L_{\mathrm{s}}(n, t)=\frac{\mathrm{I}}{2 \sqrt{2}} \int_{0}^{\pi} \cos ^{2 n-1} \frac{\psi}{2} \frac{d u}{\sqrt{z-u}}, \quad L_{2}(n, t)=\frac{\mathrm{I}}{2 \sqrt{2}} \int_{0}^{1} \cos ^{2 n-1} \frac{\psi}{2} \frac{d u}{\sqrt{u+z}}
\end{aligned}
$$


et en opérant la différentiation comme plus haut, on trouve

$$
\begin{aligned}
\frac{d L_{1}(n, t)}{d t} & =-\frac{\mathrm{I}}{2 \sqrt{2}} \sin t \frac{d}{d z} \int_{0}^{z} \cos ^{2 n-1} \frac{\psi}{2} \frac{d u}{\sqrt{z-u}} \\
& =-\frac{\mathrm{I}}{2 \sqrt{2}} \cos ^{2 n-1} \frac{\pi}{4} \frac{\sin t}{\sqrt{\cos t}}-\frac{2 n-\mathrm{I}}{4} \sin t L_{\mathrm{1}}(n-\mathrm{I}, t) \\
\frac{d L_{2}(n, t)}{d t} & =-\frac{\mathrm{I}}{2 \sqrt{2}} \sin t \frac{d}{d z} \int_{0}^{\mathrm{I}} \cos ^{2 n-1} \frac{\psi}{2} \frac{d u}{\sqrt{z+u}} \\
& =\frac{\mathrm{I}}{2 \sqrt{2}} \cos ^{2 n-1} \frac{\pi}{4} \frac{\sin t}{\sqrt{\cos t}}-\frac{2 n-\mathrm{I}}{4} \sin t L_{2}(n-\mathrm{I}, t)
\end{aligned}
$$

et par addition, on retrouve (25).

$L(n, t)$ étant essentiellement positif, nous voyons par $(25)$ que

$$
\frac{d L(n, t)}{d t}<\mathrm{o} \quad(\mathrm{o}<t<\pi)
$$

Il résulte, sans autre des relations (19), (20), que dans tout intervalle $(\varepsilon, \pi), \varepsilon>0$, on a uniformément en $t$

$$
k_{n} \frac{d L(n, t)}{d t}=O\left(n^{\frac{5}{2}} e^{-\frac{n \varepsilon^{2}}{4}}\right) \quad(0<\varepsilon \leqslant t \leqslant \pi)
$$

Nous avons encore

$$
\lim _{n=\infty} \frac{k_{n}}{2} \int_{0}^{\varepsilon} \sin ^{2} t \frac{d L(n, t)}{d t} d t=-\mathrm{I} .
$$

En effet, une intégration par parties donne pour la valeur de l'intégrale

$$
\frac{k_{n}}{2}\left|\sin ^{2} t L(n, t)\right|_{t=0}^{l=\varepsilon}-k_{n} \int_{0}^{\varepsilon} \cos t . L(n, t) \sin t d t .
$$

Le premier terme tend vers o avec $-\frac{\mathrm{I}}{n}$; quant a l'intégrale

$$
-k_{n} \int_{0}^{\varepsilon} \cos t L(n, t) \sin t d t
$$

elle converge bien d'après $\left(23_{a}\right)$ et $\left(24_{a}\right)$ vers $-\cos 0=-\mathrm{I}$.

8. Théorème général sur la limite de la suite $S_{n}(\exists, \varphi)$. Nous supposons encore que le point $(\exists, \varphi)$ se trouve au pôle. Partons de la relation $\left(23_{a}\right)$

$$
\lim _{n=\infty} S_{n}=\lim _{n=\infty} k_{n} \int_{0}^{\varepsilon} f(t) L(n, t) \sin t d t
$$

et intégrons par parties l'intégrale $\mathrm{du}$ second membre

$$
\begin{gathered}
k_{n} \int_{0}^{\varepsilon} f(t) L(n, t) \sin t d t \\
=k_{n} \int_{0}^{\varepsilon} f(t) d t \cdot \sin \varepsilon L(n, \varepsilon)-k_{n} \int_{0}^{\varepsilon} \int_{0}^{t} f(\zeta) d \xi \frac{d}{d t}[L(n, t) \sin t] d t .
\end{gathered}
$$

Le premier terme tendant vers zéro avec $\frac{I}{n}$, tout revient à calculer la limite du 
second terme, qui, développé, s’écrit

$-k_{n} \int_{0}^{\varepsilon}\left[\frac{\mathrm{r}}{\sin t} \int_{0}^{t} f(\xi) d \xi\right] \cos t L(n, t) \sin t d t-k_{n} \int_{0}^{\varepsilon}\left[-\frac{\mathrm{I}}{\sin t} \int_{0}^{t} f(\xi) d \xi\right] \sin ^{2} t \frac{d L(n, t)}{d t} d t$.

Supposons maintenant que la limite

$$
\lim _{t=+0} \frac{1}{\sin t} \int_{0}^{t} f(\xi) d \xi=\bar{f}
$$

existe et soit finie. Dans ce cas, le premier terme de la dernière expression a pour limite, d'après le $\$ 6$,

Quant au second terme, l'emploi du théorème de la moyenne (applicable puisque $\frac{d L(n, t)}{d t}$ est de signe constant) et de la relation (28) donne pour la limite de ce terme

Il vient donc

$$
+2 \vec{f}
$$

d'où le théorème:

$$
\lim _{n=\infty} S_{n}=-\bar{f}+2 \bar{f}=\bar{f}
$$

Théréme $\mathrm{V}_{a}$. - La suite $S_{n}(\pi, p)$ converge vers $\lim _{t=+\infty} \frac{\mathrm{I}}{\sin t} \int_{0}^{t} F(\tau, p ; t)$ en tout point où cette limite existe.

$\mathrm{Si}$, plus haut, nous avions intégré par parties comme suit

$$
\begin{gathered}
k_{n} \int_{0}^{\varepsilon} f(t) L(n, t) \sin t d t \\
=k_{n} \int_{0}^{\varepsilon} f(t) \sin t d t . L(n, \varepsilon)-k_{n} \int_{0}^{\varepsilon}\left[\frac{\mathrm{I}}{\sin ^{2} t} \int_{0}^{t} f(\zeta) \sin \xi d \xi\right] \frac{d L(n, t)}{d t} \sin t d t,
\end{gathered}
$$

il se serait introduit, en remplaçant $f(t)$ par sa définition $\left(25_{n}\right)$, la linite pour $t=-$-o de

et, en remarquant que

$$
\frac{I}{\sin ^{2} t} \int_{(\omega \leq n)} F\left(\vartheta^{\prime}, \varphi^{\prime}\right) d \sigma^{\prime}
$$

$$
2 \pi(1-\cos t)=4 \pi \sin ^{2} \frac{t}{2}
$$

n'est autre chose que l'aire de la calotte de centre $(\pi, \varphi)$ et de rayon sphérique égal a $t$, on obtiendrait le théorème:

Théorème $\mathrm{V}_{b}$. - La suite $S_{n}(J, \varphi)$ converge vers

$$
\lim _{i=+0} \frac{I}{2 \pi(I-\cos t)} \int_{(\omega \leq n)} F\left(\tau^{\prime}, \varphi^{\prime}\right) d \sigma^{\prime}
$$

en tout point où cette limite existe. En particulier, clle converge vers $F(x, \uparrow)$ in tout point où $F$ est égale à la dérivée de surface de son integrale superficielle, c'est-à-dire en tout point où $F$ est égale à la limite ci-dessus indiquée.

Il résulte du théorème $V$ et des propriétés des intégrales de Riemann et de Lebesgue, que la limite de $S_{n}(\tau, \rho)$ existe presque partout; les points où cette limite 
n'existe pas ou n'est pas égale à $F(\tau, \uparrow)$ forment un ensemble de mesure superficielle nulle.

\section{Chapitre II.}

\section{La sommation des dérivées.}

9. La dérivée première de $S_{n}(\exists, \uparrow)$. - Un demi grand cercle de la sphère, ayant son origine au point $(I, \varphi)$, est complètement déterminé par sa direction en ce point. $S_{n}(\pi, \varphi)$ étant une fonction continue, différentiable, la limite de

$$
\frac{S_{n}\left(\Xi^{\prime}, \varphi^{\prime}\right)-S_{n}(\Xi, \varphi)}{()}
$$

lorsque le point $\left(\jmath^{\prime}, \varphi^{\prime}\right)$ tend vers le point $(\tau, \varphi$ ) en se déplaçant sur le demi grand cercle de direction $\theta$, existe et définit la dérivée de $S_{n}(\tau, \varphi)$ suivant l'arc $\theta$

De la formule (I6)

$$
\frac{\partial S_{n}(\nexists, \varphi)}{\partial \theta}
$$

découle

$$
S_{n}(\Im, \varphi)=\frac{k_{n}}{2 \pi} \int F\left(\Im^{\prime}, \varphi^{\prime}\right) L(n, \omega) d \sigma^{\prime}
$$

$$
\begin{aligned}
\frac{\partial S_{n}(\tau, \varphi)}{\partial \theta} & =\frac{k_{n}}{2 \pi} \int F\left(\Xi^{\prime}, \varphi^{\prime}\right) \frac{\partial L(n, \omega)}{\partial \theta} d \sigma^{\prime} \\
& =\frac{k_{n}}{2 \pi} \int F\left(\Xi^{\prime}, \varphi^{\prime}\right) \frac{\partial \omega}{\partial \theta} \frac{d L(n, \omega)}{d \omega} d \sigma^{\prime} .
\end{aligned}
$$

Du théorème connu que dans un triangle sphérique un côté est plus grand que la différence des deux autres, résulte

$$
\left|\frac{\partial \omega}{\partial \theta}\right| \leq \mathrm{r}
$$

Il suffira donc, puisque alors (27)

$$
\left|\frac{k_{n}}{2 \pi} \int_{(\omega \supseteq \varepsilon)} F\left(\jmath^{\prime}, \varphi^{\prime}\right) \frac{\partial \omega}{\partial \theta} \frac{d L(n, \omega)}{d \omega} d \sigma^{\prime}\right| \leq O\left(n^{\frac{5}{2}} e^{-\frac{n \varepsilon^{2}}{4}}\right) \int\left|F\left(\jmath^{\prime}, \varphi^{\prime}\right)\right| d \sigma^{\prime},
$$

d'étudier la limite

$$
\lim _{n=\infty} \frac{\partial S_{n}(\tau, \varphi)}{\partial \theta}=\lim _{n=\infty} \frac{k}{2 \pi} \int_{(\omega \leq \varepsilon)} F\left(\Im^{\prime}, \varphi^{\prime}\right) \frac{\partial \omega}{\partial \theta} \frac{d L(n, \omega)}{d \omega} d \sigma^{\prime},
$$

donc:

ThÉORĖME VI. - La convergence en un point de la stite $\frac{\partial S_{n}(\nexists, p)}{\partial \theta}$ ne dépend que des valeurs de la fonction $F$ dans le voisinage de ce point.

Par suite de l'indépendance des quantités qui interviennent dans la dernière formule de la position du système de coordonnées, nous nous bornerons à placer le point $(\jmath, \varphi)$ considéré au pôle. Nous prenons donc $z=0, \omega=J^{\prime}$. Le demi grand cercle $\theta$ 
est maintenant un méridien de longitude $\varphi \cdot \frac{\partial \omega}{\partial \theta}$ se réduit alors à $\left(\frac{\partial \omega}{\partial \Im}\right)_{9=0}$. Or de

$$
\cos \omega=\cos \approx \cos \beth^{\prime}+\sin \Im \sin \Im^{\prime} \cos \left(\varphi-\varphi^{\prime}\right)
$$

vient en dérivant par rapport $\dot{d} \dot{\delta}, \varphi$ restant fixe,

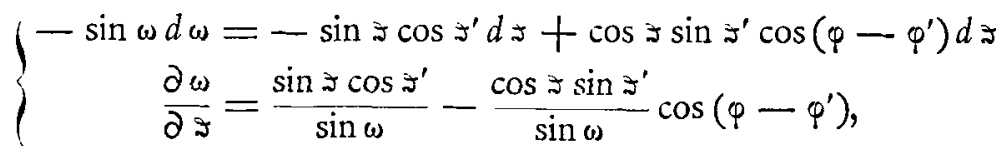

d'où, pour $\Im=0, \omega=s^{\prime}$,

On a donc

$$
\left(\frac{\partial \omega}{\partial a}\right)_{g=0}=-\cos \left(\varphi-\varphi^{\prime}\right) .
$$

$$
\lim _{n=\infty} \frac{\partial S_{n}(0, \varphi)}{\partial \jmath}=-\lim _{n=\infty} \frac{k_{n}}{2 \pi} \int_{0}^{\varepsilon} d \jmath^{\prime} \sin \jmath^{\prime} \frac{d L\left(n, \jmath^{\prime}\right)}{d \jmath^{\prime}} \int_{0}^{2 \pi} F\left(\jmath^{\prime}, \varphi^{\prime}\right) \cos \left(\varphi-\varphi^{\prime}\right) d \varphi^{\prime} .
$$

Notons

Il vient

$$
\frac{\mathrm{I}}{\pi \sin \Im^{\prime}} \int_{0}^{2 \pi} F\left(\Im^{\prime}, \varphi^{\prime}\right) \cos \left(\varphi-\varphi^{\prime}\right) d \varphi^{\prime}=F_{1}\left(\Im^{\prime}, \varphi\right) \text {. }
$$

$$
\lim _{n=\infty} \frac{\partial S_{n}(0, \varphi)}{\partial \succsim}=-\lim _{n=\infty} \frac{k_{n}}{2} \int_{0}^{\varepsilon} F_{1}(t, \varphi) \sin ^{2} t \frac{d L(n, t)}{d t} d t .
$$

$\lambda$ étant une valeur moyenne de $F_{1}(t, \varphi)$ dans $o<t<\varepsilon$, le théorème de la moyenne donne pour la dernière intégrale

$$
-\lambda \frac{k_{n}}{2} \int_{0}^{\varepsilon} \sin ^{2} t \frac{d L(n, t)}{d t} d t
$$

Si donc $F_{\mathrm{r}}(t, \varphi)$ a une limite $F_{\mathrm{r}}(+0, \varphi)$ pour $t=+0$, on aura (28)

La limite

$$
\lim _{n=x} \frac{\partial}{\partial g} S_{n}(0, \varphi)=F_{s}(+o, \varphi) \text {. }
$$

$$
\lim _{t=+0} \frac{\mathrm{I}}{\pi \sin t} \int_{0}^{2 \pi} F\left(t, \varphi^{\prime}\right) \cos \left(\varphi-\varphi^{\prime}\right) d \varphi^{\prime}
$$

est dite, par analogie, la dérivée généralisée au pôle $\approx=0$ de la fonction $F(\nexists, \varphi)$ relativement au méridien de longitude $p$. On la définit sans difficulté en un point quelconque pour une direction quelconque et l'on peut énoncer le résultat:

THÉORÈME VII. - La suite

$$
\frac{\partial}{\partial \theta} S_{n}(\tau, \varphi)=\sum_{k=1}^{n} \frac{n(n-1) \ldots(n-k+1)}{(n+2)(n+3) \ldots(n+k+1)} \frac{2 k+1}{4 \pi} \frac{\partial}{\partial \theta} \int F\left(z^{\prime}, \varphi^{\prime}\right) P_{k}(\cos \omega) d \sigma^{\prime}
$$
a pour limite la dérivée généralisée de la fonction $F(\xi, \varphi)$ suivant l'arc $\theta$ au point $(\tau, \varphi)$, lorsque cette dérivée généralisée existe.

Il résulte encore de la démonstration que $\frac{\partial}{\partial \tau} S_{n}(0, p)$ a ses limites inférieure et supérieure pour $n=\infty$ comprises entre les limites inférieure et supérieure de

$$
\frac{I}{\pi \sin t} \int_{0}^{2 \pi} F\left(t, \varphi^{\prime}\right) \cos \left(\varphi-\varphi^{\prime}\right) d \varphi^{\prime} \quad(0<t \leqslant \varepsilon) .
$$

Rend. Circ. Matem. Palermo, t. XXXIII ( ${ }^{\circ}$ sem. 1912 ). - Stampato il 4 gennajo 1912. 
Dans l'hémisphère nord, $F\left(\Im^{\prime}, \varphi^{\prime}\right)$ est une fonction univoque des deux variables

$$
x=\sin z^{\prime} \cos \varphi^{\prime}, \quad y=\sin \tau^{\prime} \sin \varphi^{\prime},
$$

le point $(x, y)$ étant la projection du point $\left(x^{\prime}, \varphi^{\prime}\right)$ sur le plan de l'équateur; soit

$$
F(z, p)=\mathfrak{F}(x, y) \text {. }
$$

Si $\mathfrak{F}(x, y)$ possède au point $x=0, y=0$ une différentielle première totale, c'est-à-dire:

$\mathrm{I}^{\circ}$ si $\frac{\partial \mathfrak{F}}{\partial x}, \frac{\partial \mathfrak{F}}{\partial y}$ existent et sont finies au point $x=0, y=0$,

$2^{\circ}$ si dans le voisinage du point $x=0, y=0$ la fonction peut s'exprimer par

$$
\mathfrak{F}(x, y)=\mathfrak{F}(\mathrm{o}, 0)+x \frac{\partial \mathfrak{F}(\mathrm{o}, \mathrm{o})}{\partial x}+\frac{\partial \mathfrak{F}(\mathrm{o}, \mathrm{o})}{\partial y}+x n_{1}+y n_{2}
$$

aves

$$
\lim _{x=0, y=0} x_{1}=\lim _{x=0, y=0} x_{y_{2}}=0
$$

quand $x, y$ tendent indépendamment vers zéro, nous dirons, pour abréger, que $F(z, \varphi)$ possède au pale une différentielle premìre. Dans ce cas, dans le voisinage du pôle,

$$
F\left(\Im^{\prime}, p^{\prime}\right)=\mathfrak{F}(0,0)+\sin s^{\prime}\left[\frac{\partial \mathfrak{F}(0, o)}{\partial x} \cos \varphi^{\prime}+\frac{\partial \mathfrak{F}(o, o)}{\partial y^{\prime}} \sin \varphi^{\prime}\right]+\sin x^{\prime} \cdot n_{3}
$$

Il vient donc

$$
\lim _{y^{\prime}=0} \max _{0}\left|n_{j}\right|=0
$$

$$
\begin{gathered}
\int_{0}^{2 \pi} F\left(\jmath^{\prime}, \varphi^{\prime}\right) \cos \left(\varphi-\varphi^{\prime}\right) d \varphi^{\prime}=\pi \sin J^{\prime}\left[\frac{\partial \mathfrak{F}(0,0)}{\partial x} \cos \varphi+\frac{\partial \mathfrak{F}(0,0)}{\partial y} \sin \varphi\right] \\
+\sin \jmath^{\prime} \int_{0}^{2 \pi} \pi_{i} \cos \left(\varphi-\varphi^{\prime}\right) d \varphi^{\prime}
\end{gathered}
$$

d'où

$$
\lim _{\gamma^{\prime}=0} \frac{I}{\pi \sin \tau^{\prime}} \int_{0}^{2 \pi} F\left(\tau^{\prime}, \varphi^{\prime}\right) \cos \left(p-p^{\prime}\right) d p^{\prime}=\frac{\partial \mathfrak{F}(0,0)}{\partial x} \cos p+\frac{\partial \mathfrak{F}(0,0)}{\partial y} \sin p \text {. }
$$

Mais comme le second membre n'est autre que la dírivée de $F$ relativement à l'arc de longitude $q$ au pôle, nous voyons qu'en tout point où $F(J, ?)$ possède une ditférentielle première, la dérivée généralisée de $F$ relativement à un arc $\theta$ passant par ce point est égale à la dérivée $\frac{\partial F}{\partial \theta}$. Le thcorcine VII a donc le

Corollaire. - La suite $\frac{\partial}{\partial G} S_{n}(\tau, \varphi)$ a pour limite $\frac{\partial F(\tau, \varphi)}{\partial \xi}$, en tout point oit $F$ possède une différentielle premièré.

$s=s(u, v), \varphi=\varphi(u, v)$ étant une représentation paramétrique régulière d'une calotte sphérique, dans laquelle $F$ possède une différentielle et où $\frac{\partial F}{\partial u}$ est continue, convergence de la suite $\frac{\partial}{\partial u} S_{n}(\jmath, \psi)$ vers $\frac{\partial F}{\partial u}$ est uniforme dans toute calotte entièrement intérieure à la première. C'est immédiat.

I0. La limite de la dérivée seconde $\frac{\partial^{2}}{\partial \theta^{2}} S_{u}(\tau, p) .-\theta_{1}$ et $\theta_{2}$ étant les arcs comptés sur deux grands cercles de plans perpendiculaires passant par le point $(\tau, p)$, les dé- 
rivées secondes à étudier sont au nombre de trois,

$$
\frac{\partial^{2} S_{n}}{\partial \theta_{1}^{2}}, \quad \frac{\partial^{2} S_{n}}{\partial \theta_{1} \partial \hat{\theta}_{2}^{-}}, \quad \frac{\partial^{2} S_{n}}{\partial \theta_{2}^{2}}
$$

L'étude de la troisième se ramène évidemment à l'étude de la première. Consacrons ce $\int$ à la première. De la valeur donnée au $\int 9$ pour $\frac{\partial}{\partial \theta} S_{n}(\Omega, \varphi)$ découle, par une nouvelle diffesentiation,

$$
\begin{aligned}
\frac{\partial^{2}}{\partial \theta^{2}} S_{n}(\tau, \varphi) & =\frac{k_{n}}{2 \pi} \int F\left(\sigma^{\prime}, \varphi^{\prime}\right) \frac{\partial}{\left.\partial{ }^{\prime}\right)}\left[\frac{\partial \omega}{\partial \theta} \frac{d L(n, \omega)}{d \omega}\right] d \sigma^{\prime} \\
& =\frac{k_{n}}{2 \pi} \int F\left(\sigma^{\prime}, \varphi^{\prime}\right) \frac{\partial^{2} \omega}{\partial \xi^{\prime}} \frac{d L(n, \omega)}{d \omega} d \sigma^{\prime}+\frac{k}{2 \pi} \int F\left(\sigma^{\prime}, \varphi^{\prime}\right)\left(\frac{\partial \omega}{\partial \xi}\right)^{2} \frac{d^{2} L(n, \omega)}{d \omega^{2}} d \sigma^{\prime} .
\end{aligned}
$$

Si nous prenons pour $F$ la valeur

$$
F\left(\Im^{\prime}, \varphi^{\prime}\right)=\mathrm{I}
$$

$S_{n}(\tau, \eta)$ se réduit alors d̀ I et $\frac{\partial^{2}}{\partial \theta^{2}} S_{n}(\tau, \varphi)$ à o, donc

$$
\frac{k_{n}}{2 \pi} \int \frac{\partial}{\partial \theta}\left[\frac{\partial \omega}{\partial \dot{H}} \frac{d L(n, \omega)}{d(\omega)}\right] d \sigma^{\prime}=\mathrm{o} .
$$

Si nous supposons que la valeur moyenne $F(\mathrm{~J}, \rho ;+0)$ existe au point considéré, nous aurons, en retranchant de la précédente la dernière relation multipliée par $F(\jmath, \varphi ;+0)$,

$$
\begin{gathered}
\frac{\partial^{2}}{\partial \theta^{2}} S_{n}(\tau, \varphi)=\frac{k_{n}}{2 \pi} \int\left[F\left(\Im^{\prime}, \vartheta^{\prime}\right)-F(\tau, \uparrow ;+0)\right] \frac{\partial^{2} \omega}{\partial \theta^{2}} \frac{d L(n, \omega)}{\partial \omega} d \sigma^{\prime} \\
+\frac{k_{n}}{2 \pi} \int\left[F\left(\tau^{\prime}, \varphi^{\prime}\right)-F(\tau, \uparrow ;+0)\right]\left(\frac{\partial \omega}{\partial \theta}\right)^{2} \frac{d^{2} L(n, \omega)}{d \omega^{2}} d \sigma^{\prime} .
\end{gathered}
$$

Prenons maintenant, pour simplifier, le point $(\tau, \phi)$ au pôle et soit $\varphi$ la longitude de l'arc $\theta$; il nous suffira de chercher la limite de

Nous avons déjà trouvé (3I)

$$
\frac{\partial^{2}}{\partial \bar{j}^{2}} S_{n}(0, \varphi)
$$

$$
\left(\frac{\partial \omega}{\partial \jmath}\right)_{g=0}=-\cos \left(\varphi-\varphi^{\prime}\right)
$$

En différentiant encore une fois la formule (30) par rapport à $\Xi$

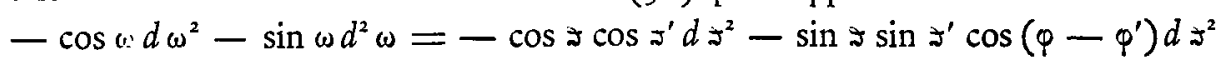

il vient

$$
\frac{\partial^{2} \omega}{\partial \tau^{2}}=-\frac{\cos \omega}{\sin \omega}\left(\frac{\partial \omega}{\partial \jmath}\right)^{2}+\frac{\cos \tau \cos \jmath^{\prime}}{\sin \omega}+\frac{\sin \ni \sin \jmath^{\prime}}{\sin \omega} \cos \left(\varphi-\varphi^{\prime}\right)
$$

et pour $\mathrm{z}=0$

$$
\left(\frac{\partial^{2} \omega}{\partial \partial^{2}}\right)_{\Im=0}=\operatorname{cotg} \nexists^{\prime} \sin ^{2}\left(\varphi-\varphi^{\prime}\right) \text {. }
$$


Done

$$
\begin{aligned}
\frac{\partial^{2}}{\partial J^{2}} S_{n}(0, \varphi) & =\frac{k_{n}}{2 \pi} \int\left[F\left(\Im^{\prime}, \varphi^{\prime}\right)-f(+o)\right] \operatorname{cotg} \Im^{\prime} \cdot \sin ^{2}\left(\varphi-\varphi^{\prime}\right) \frac{d L\left(n, \jmath^{\prime}\right)}{d \jmath^{\prime}} d \sigma^{\prime} \\
& +\frac{k_{n}}{2 \pi} \int\left[F\left(\jmath^{\prime}, \varphi^{\prime}\right)-f(+0)\right] \cos ^{2}\left(\varphi-\varphi^{\prime}\right) \frac{d^{2} L\left(n, J^{\prime}\right)}{\partial J^{\prime 2}} d \sigma^{\prime},
\end{aligned}
$$

en notant par $f(+0)$ la valeur moyenne supposée existante au pôle. Notons

Alors

$$
\begin{aligned}
& g(t, \varphi)=\frac{\mathrm{I}}{\pi \sin ^{2} t} \int_{0}^{2 \pi}\left[F\left(t, \varphi^{\prime}\right)-f(+0)\right] \sin ^{2}\left(\varphi-\varphi^{\prime}\right) d \varphi^{\prime}, \\
& h(t, \varphi)=\frac{\mathrm{I}}{\pi \sin ^{2} t} \int_{0}^{2 \pi}\left[F\left(t, \varphi^{\prime}\right)-f(+0)\right] \cos ^{2}\left(\varphi-\varphi^{\prime}\right) d \varphi^{\prime} .
\end{aligned}
$$

$\frac{\partial^{2}}{\partial \hbar^{2}} S_{n}(0, \psi)=\frac{k_{n}}{2} \int_{0}^{\pi} g(t, \Psi) \cos t \cdot \sin ^{2} t \frac{d L(n, t)}{d t} d t+\frac{k_{n}}{2} \int_{0}^{\pi} h(t, \varphi) \cdot \sin ^{3} t \frac{d^{2} L(n, t)}{d t^{2}} d t$

Si donc

$$
\lim _{t=+0} g(t, p)=g(+o, p)
$$

existe, nous aurons immédiatement, d'après une recherche déjà faite au $₫ 8$, pour la limite du premier terme la valeur

$$
-g(+o, \varphi)
$$

Pour calculer la limite du second terme, rematquons qu'à teneur de (25)

$$
\left\{\begin{aligned}
\frac{d^{2} L(n, t)}{d t^{2}} & =-\frac{2 n-\mathrm{I}}{4} \cos t L(n-\mathrm{I}, t)-\frac{2 n-\mathrm{I}}{4} \sin t \frac{d L(n-\mathrm{I}, t)}{d t} \\
& =\frac{\cos t}{\sin t} \frac{d L(n, t)}{d t}-\frac{2 n-\mathrm{I}}{4} \sin t \frac{d L(n-\mathrm{I}, t)}{d t} .
\end{aligned}\right.
$$

On a donc, uniformément en $t$, dans tout intervalle $(\varepsilon, \pi), \varepsilon>0$, d'après (20) et (27),

$$
k_{n} \frac{d^{2} L(n, t)}{d t^{2}}=O\left(n^{\frac{7}{2}} e^{-\frac{n \varepsilon^{2}}{4}}\right)
$$

$$
(0<\varepsilon \leqslant t \leqslant \pi)
$$

La limite du second terme est donc la même que celle de

$$
\begin{gathered}
\frac{k_{n}}{2} \int_{0}^{\varepsilon} b(t, \varphi) \sin ^{3} t \frac{d^{2} L(n, t)}{d t^{2}} d t \\
=\frac{k_{n}}{2} \int_{0}^{\varepsilon} h(t, \varphi) \cos t \cdot \sin ^{2} t \frac{d L(n, t)}{d t} d t-\frac{2 n-\mathrm{I}}{4} \frac{k_{n}}{2} \int_{0}^{\varepsilon} h(t, \varphi) \sin ^{4} t \frac{d L(n-\mathrm{I}, t)}{d t} d t .
\end{gathered}
$$

Or, si la limite

$$
\lim _{t=+0} b(t, \varphi)=b(+o, \varphi)
$$

existe, au second membre le premier terme a pour limite, d'après les calculs faits au $\ 8$,

$$
-b(+o, \varphi) \text {. }
$$

Quant au second terme, sa valeur est, d'après le théorème de la moyenne,

$$
-\frac{2 n-\mathrm{I}}{4} \frac{k_{n}}{2} \bar{b} \int_{0}^{\varepsilon} \sin ^{4} t \frac{d L(n-\mathrm{I}, t)}{d t} d t
$$


$\bar{b}$ étant une valeur moyenne de $h(t, \varphi)$ dans $o<t<\varepsilon$. En intégrant par parties

$$
\begin{gathered}
-\frac{2 n-\mathrm{I}}{4} \frac{k_{n}}{2} \int_{0}^{\varepsilon} \sin ^{4} t \frac{d L(n-\mathrm{I}, t)}{d t} d t=-\frac{2 n-\mathrm{I}}{4} \frac{k_{n}}{2} \sin ^{4} \varepsilon L(n-\mathrm{I}, \varepsilon) \\
+2 k_{n} \int_{0}^{\varepsilon} \sin ^{3} t \cos t \frac{2 n-\mathrm{I}}{4} L(n-\mathrm{I}, t) d t,
\end{gathered}
$$

le premier terme tend vers zéro et le second est égal, d'après (25), à

$$
2 k_{n} \int_{0}^{\varepsilon} \cos t \sin ^{2} t \frac{d L(n, t)}{d t} d t .
$$

Il a donc 4 pour limite, d'après (28). L'intégrale considérée a donc la limite

$$
4 b(+o, \varphi) \text {. }
$$

Rassemblant les résultats obtenus, on voit que

$$
\begin{aligned}
\lim _{n=\infty} \frac{\partial^{2}}{\partial \jmath^{2}} S_{n}(0, \varphi) & =-g(+0, \varphi)-b(+0, \varphi)+4 b(+0, \varphi) \\
& =3 h(+0, \varphi)-g(+0, \varphi)
\end{aligned}
$$

lorsque ces quantités existent. La valeur

$$
\begin{aligned}
3 h(+o, \varphi)-g(+o, \varphi) & =\frac{3}{\pi} \lim _{t=+0} \frac{I}{\sin ^{2} t} \int_{0}^{2 \pi}\left[F\left(t, \varphi^{\prime}\right)-f(+0)\right] \cos ^{2}\left(\varphi-\varphi^{\prime}\right) d \varphi^{\prime} \\
& -\frac{1}{\pi} \lim _{t=+0} \frac{I}{\sin ^{2} t} \int_{0}^{2 \pi}\left[F\left(t, \varphi^{\prime}\right)-f(+0)\right] \sin ^{2}\left(\varphi-\varphi^{\prime}\right) d \varphi^{\prime}
\end{aligned}
$$

peut être appelée, lorsqu'elle existe, la dérivée seconde généralisée de la fonction $F$ au pôle, relativement à l'arc de longitude $\varphi \cdot f(+0)$ y désigne la valeur moyenne supposée existante de $F$ au pôle.

En notant comme plus haut $(33) F\left(J^{\prime}, \varphi^{\prime}\right)=\mathfrak{F}(x, y)$ et en supposant que $\mathfrak{F}$ possède au point $x=0, y=0$ une différentielle seconde, on verrait que la dérivée généralisée est dans ce cas égale ì la valeur de la dérivée seconde ordinaire. En particulier, pour $\varphi=0$, on trouve

d'où

$$
\begin{aligned}
& h(+o, \varphi)=\frac{I}{8}\left[3 \frac{\partial^{2} \mathfrak{F}(0,0)}{\partial x^{2}}+\frac{\partial^{2} \mathfrak{F}(0,0)}{\partial y^{2}}\right], \\
& g(+o, \varphi)=\frac{I}{8}\left[\frac{\partial^{2} \mathfrak{F}(0,0)}{\partial x^{2}}+3 \frac{\partial^{2} \mathfrak{F}(0,0)}{\partial y^{2}}\right],
\end{aligned}
$$

$$
3 b(+0, \varphi)-g(+0, \varphi)=\frac{\partial^{2} \mathfrak{F}(0,0)}{\partial x^{2}} \text {. }
$$

Nous pouvons donc énoncer le théorème:

THÉORÉME VIII. - La suite $\frac{\partial^{2}}{\partial \theta^{2}} S_{n}(\exists, \varphi)$ converge en tout point $(\exists, \varphi)$ où la fonction $F$ possède une dérivée seconde généralisée relativement à l'arc $\theta$ vers la valeur de cette dérivée. En particulier, elle converge vers $\frac{\partial^{2} F(\tau, \varphi)}{\partial \theta^{2}}$ en tout point ou $F$ possède une différentielle seconde totale. 
I I. La suite $\frac{\partial^{2}}{\partial \theta_{1} \partial \theta_{2}} S_{n}(\Xi, \varphi)$ - - $\theta_{1}$ et $\theta_{2}$ désignant les ares pris sur deux grands cercles perpendiculaires, on a $\frac{\partial^{2}}{\partial \theta_{1} \partial \theta_{2}} S_{n}(\exists, p)=\frac{k_{n}}{2 \pi} \int F\left(J^{\prime}, p^{\prime}\right) \frac{\partial^{2} \omega}{\partial \theta_{1} \partial \theta_{2}} \frac{d L(n, \omega)}{d \omega} d \sigma^{\prime}+\frac{k_{n}}{2 \pi} \int F\left(J^{\prime}, \stackrel{\prime}{\prime}^{\prime}\right) \frac{\partial \omega}{\partial \theta_{1}} \frac{\partial \omega}{\partial \theta_{2}} \frac{d^{2} L(n, \omega)}{\left.d \omega^{2}\right)^{2}} d \sigma^{\prime}$. $\frac{\partial^{2} \omega}{\partial \theta_{s} \partial \theta_{2}}$ et $\frac{\partial \omega}{\partial \theta_{1}}, \frac{\partial \omega}{\partial \theta_{2}}$ étant bornés dans toute calotte $\omega \triangleq \varepsilon$, on voit, comme plus haut, qu'il suffit de considérer les intégrales étendues à la calotte $\omega \leqslant \varepsilon$. Donc,

$$
\begin{aligned}
& \lim _{n=x} \frac{\partial^{2}}{\partial \theta_{1} \partial \theta_{2}} S_{n}(J, \psi)
\end{aligned}
$$

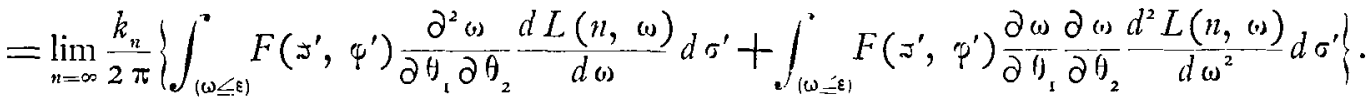

On pourrait tirer de là un théorème analogue au théorème VI.

Prenons maintenant, pour simplifier, $(\exists, \psi)$ au pôle et comme direction de $\theta_{1}$ et de $\theta_{2}$ les directions $\psi=0$ et $\psi=\frac{\pi}{2}$. Pour calculer rapidement les valeurs $\frac{\partial \omega}{\partial \theta_{1}}$, $\frac{\partial(\omega)}{\partial \theta_{2}}, \frac{\partial^{2} \omega}{\partial \theta_{1} \partial \theta_{2}}$, posons

$$
x=\sin \ni \cos \varphi, \quad y=\sin \ni \sin \varphi ; \quad x^{\prime}=\sin \star^{\prime} \cos \psi^{\prime}, \quad y^{\prime}=\sin \Im^{\prime} \sin \varphi^{\prime} .
$$

Il en résulte, dans le voisinage du pôle, à des infiniments petits d'ordre supérieur près,

$$
\left\{\begin{array}{c}
\omega=\sqrt{\left(x-x^{\prime}\right)^{2}+\left(y-y^{\prime}\right)^{2}}, \\
\frac{\partial \omega}{\partial \theta_{1}}=\left(\frac{\partial \omega}{\partial x}\right)_{x=y=0}=-\frac{x^{\prime}}{\sqrt{x^{\prime 2}+y^{\prime 2}}}=-\cos \varphi^{\prime}, \\
\frac{\partial \omega}{\partial \theta_{2}}=\left(\frac{\partial \omega}{\partial y}\right)_{x=y=0}=-\frac{y^{\prime}}{1^{\prime} x^{\prime 2}+y^{\prime 2}}=-\sin \varphi^{\prime}, \\
\frac{\partial^{2} \omega}{\partial \theta_{1} \partial_{2} \theta_{2}}=\left(\frac{\partial^{2} \omega}{\partial x} \frac{x^{\prime} y^{\prime}}{\partial y}\right)_{x=y=0}=-\frac{\sin \varphi^{\prime} \cos \varphi^{\prime}}{\sin \Im^{\prime}}
\end{array}\right.
$$

On aura donc à étudier au pôle la limite de

$-\frac{k_{n}}{2 \pi} \int_{(\omega \leq \varepsilon)} F\left(J^{\prime}, \rho^{\prime}\right) \frac{\sin \varphi^{\prime} \cos \varphi^{\prime}}{\sin \tau^{\prime}} \frac{d L\left(n, \tau^{\prime}\right)}{d \tau^{\prime}} d \sigma^{\prime}+\frac{k_{n}}{2 \pi} \int_{(\omega \leq \varepsilon)} F\left(\tau^{\prime}, \varphi^{\prime}\right) \sin \varphi^{\prime} \cos \varphi^{\prime} \frac{d^{2} L\left(n, \Xi^{\prime}\right)}{d \tau^{\prime 2}} d \sigma^{\prime}$ ou encore, en posant

celle de

$$
l(t)=\frac{I}{\pi \sin ^{2} t} \int_{0}^{2 \pi} F\left(t, \varphi^{\prime}\right) \sin \varphi^{\prime} \cos \varphi^{\prime} d \varphi^{\prime}
$$

$$
-\frac{k_{n}}{2} \int_{0}^{\varepsilon} l(t) \sin ^{2} t \frac{d L(n, t)}{d t} d t+\frac{k_{n}}{2} \int_{0}^{\varepsilon} l(t) \sin ^{3} t \frac{d^{2} L(n, t)}{d t^{2}} d t
$$

Les analyses des $\int \mathbb{S}$ précédents montrent que, lorsque

$$
\lim _{t=+0} l(t)=l(+o)
$$

existe, les limites de ces deux intégrales sont respectivement $l(+0)$ et $3 l(+0)$. Par 
suite, pour les valeurs de $\theta_{1}$ et $\theta_{2}$ considérées

$$
\lim \frac{\partial^{2} S_{n}}{\partial \theta_{1} \partial \theta_{2}}=4 l(+o)=\lim _{t=+o} \frac{I}{\pi \sin ^{2} t} \int_{0}^{2 \pi} F\left(t, \varphi^{\prime}\right) \sin 2 \varphi^{\prime} d \varphi^{\prime} .
$$

Au cas où $F(\tau, p)$ possède au pôle une différentielle seconde totale, on voit que pour les valeurs de $\theta_{1}$ et $\theta_{2}$ considérées

$$
\left(\frac{\partial^{2} F(\tau, \varphi)}{\partial \theta_{\mathrm{I}} \partial \theta_{2}}\right)_{\xi=0}=\frac{\partial^{2} \mathfrak{F}(0,0)}{\partial x^{-} \partial y^{\prime}}=\lim _{t=+0} \frac{\mathrm{I}}{\pi \sin ^{2} t} \int_{0}^{2 \pi} F\left(t, \varphi^{\prime}\right) \sin 2 \varphi^{\prime} d \varphi^{\prime} .
$$

Appelant cette limite dérivée seconde généralisée relativement aux arcs $\theta_{1}$ et $\theta_{2}$, nous obtenons donc le

ThÉORÉME IX. - La suite $\frac{\partial^{2}}{\partial \theta_{1} \partial H_{2}} S_{n}(\pi, \varphi)$ converge vers la dérivie seconde généralisée de $F$ relativement is $\theta_{1}$ et $\theta_{2}$, en tout point ou cette dérivée existe. En particulier, elle converge vers $\frac{\partial^{2} F(\Im, \varphi)^{2}}{\partial \theta_{1} \partial \theta_{2}}$ en tout point où $F$ posséde une différentielle seconde.

12. Les dérivéss d'ordre supérieur. - On pourrait pousser plus loin l'analyse pour les dérivées d'ordre supérieur à 2. Les calculs sont compliqués, mais la marche à suivre est évidente. On pourra toujours démontrer au moyen des formules dérivées de (25) et en remarquant que les dérivées $\frac{\partial^{n_{1}+n_{2}} \omega}{\partial \theta_{1}^{n_{1}} \partial \theta_{2}^{n_{2}}}$ restent bornées dans la calotte $\omega \supseteq \varepsilon$, que la convergence des suites dérivées ne dépend que des valeurs de $F$ au voisinage du point considéré. Il s'introduira tout naturellement des dérivées généralisées qui se ramènent aux dérivées ordinaires lorsque la fonction possède une différentielle de l'ordre correspondant. On arrive ainsi au théorème suivant:

ThÉorème X. $-F(\tau, \psi)$ étant une fonction alsolument intégrable sur la splière, $\theta_{1}$ et $\theta_{2}$ les longueurs compties sur deux arcs de grand cercle orthogonaul $x$ passant par le point $(\nexists, \varphi)$, la suite

$\frac{\partial^{n_{1}+n_{2}}}{\partial \theta_{1}^{n_{1}} \partial \theta_{2}^{n_{2}}} S_{n}(\tau, \varphi)=\sum_{k=1}^{n} \frac{n(n-1) \ldots(n-k+1)}{(n+2)(n+3) \ldots(n+k+\mathrm{I})} \frac{\partial^{n_{1}+n_{2}}}{\partial \hat{H}_{1}^{n_{1}} \partial \theta_{2}^{n_{2}}} \int F\left(\tau^{\prime}, \varphi^{\prime}\right) P_{k}(\cos \omega) d \sigma^{\prime}$

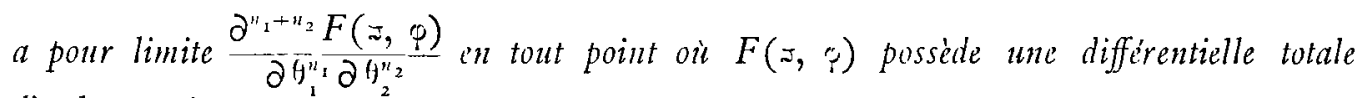
d'ordre $n_{1}+n_{2}$.

On verrait encore que lorsque dans toute une calotte, à représentation paramétrique régulière $(u, v), F(j, q)$ possède une différentielle totale d'ordre $n_{1}+n_{2}$ et une dérivée $\frac{\partial^{n_{1}+n_{2}} F(\Im, \varphi)}{\partial u^{n_{1}} \partial v^{n_{2}}}$ continue, la convergence de $\frac{\partial^{n_{1}+n_{2}}}{\partial u^{n_{1}} \partial v^{n_{2}}} S_{n}(\tau, \varphi)$ est uniforme dans toute calotte entièrement intéricure à la pricédente.

CHAPITRE III.

\section{La sommation des séries de Legendre.}

13. Un théor ’̀me général. - Des résultats précédents sur la sommation des séries de LAPLACE on passe par une méthode connue aux résultats analogues sur la somma- 
tion des séries de Legendre. Prenons, en effet, pour $F(\tau, \varphi)$ une fonction indépendante de $\varphi$

$$
F(\Im, \varphi)=F(\exists) \text {. }
$$

Le terme général de la série de Laplace de $F(\Im)$

$$
\frac{2 k+\mathrm{I}}{4 \pi} \int F\left(\pi^{\prime}\right) P_{k}(\cos \omega) d \sigma^{\prime}
$$

se réduit, par l'emploi de la formule d'addition des fonctions sphériques

$$
P_{n}(\cos \omega)=P_{n}(\cos \pi) P_{n}\left(\cos \jmath^{\prime}\right)+2 \sum_{s=1}^{n} \frac{(n-s) !}{(n+s) !} P_{n s}(\cos \jmath) P_{n s}\left(\cos \jmath^{\prime}\right) \cos s\left(\varphi-\varphi^{\prime}\right),
$$
à

$$
\frac{2 k+1}{2} P_{k}(\cos \Im) \int_{0}^{\pi} F\left(\jmath^{\prime}\right) P_{k}\left(\cos \jmath^{\prime}\right) \sin \jmath^{\prime} d \jmath^{\prime} .
$$

La série de Laplace de $F(\tau)$ est donc

$$
\frac{\mathrm{I}}{2} \int_{0}^{\pi} F\left(s^{\prime}\right) \sin z^{\prime} d z^{\prime}+\sum_{k=1}^{\infty} \frac{2 k+1}{2} P_{k}(\cos \Xi) \int_{0}^{\pi} F\left(z^{\prime}\right) P_{k}\left(\cos z^{\prime}\right) \sin z^{\prime} d s^{\prime} .
$$

Tous les théorèmes démontrés dans les deux premiers chapitres sont applicables à cette série. Introduisons

la série devient

$$
\begin{gathered}
x=\cos z \\
F(\Im)=f(x), \quad-\mathrm{I} \leq x \leq+\mathrm{I}
\end{gathered}
$$

$$
\frac{\mathrm{I}}{2} \int_{-\mathrm{I}}^{+1} f(\xi) d \xi+\sum_{k=1}^{\infty} \frac{2 k+\mathrm{I}}{2} \int_{-1}^{+\mathrm{I}} f(\xi) P_{k}(\xi) d \xi . P_{k}(x) .
$$

C'est la série de Legendre de $f(x)$. Pour pouvoir former cette série, il faut et il suffit que

$$
\int_{-1}^{+1}|f(x)| d x
$$

existe; cela revient à prendre $|F(\ni)|$ intégrable sur la sphère.

Tout point de continuité de $F(\exists)$ sur la sphère est un point de continuité pour $f(x)$ et réciproquement. $\mathrm{Si}$, en un point intérieur de l'intervalle $(-\mathrm{I},+\mathrm{I}), f(x+0)$ et $f(x-0)$ existent, $F(z+0)$ et $F(\jmath-0)$ existent et $F(\jmath)$ possède sur tout le parallèle de distance polaire $s$ une valeur moyenne, égale à

$$
\frac{F(z+0)+F(z-0)}{2}=\frac{f(x+0)+f(x-0)}{2} .
$$

En effet, fixant un point $(\tau, \varphi)$ de longitude $\rho$ quelconque, la valeur moyenne est définie par la limite pour $t=+o$ de (25)

$$
\frac{\mathrm{I}}{s(t)} \int_{\omega=t} F\left(\bar{s}^{\prime}, \varphi^{\prime}\right) d s
$$

Le parallèle passant par $(\Im, \varphi)$ coupe la circonférence $\omega=t$ en deux arcs

$$
\begin{aligned}
& \tau-t \leq s^{\prime} \leq s, \\
& s \leq s^{\prime} \leq s+t,
\end{aligned}
$$


et, $s_{1}(t)$ et $s_{2}(t)$ étant les longueurs de ces deux arcs

on a

$$
s_{1}(t)=\int_{(1)} d s, \quad s_{2}(t)=\int_{(2)} d s
$$

$$
\lim _{t=+0} \frac{s_{1}(t)}{s(t)}=\lim _{t=+0} \frac{s_{2}(t)}{s(t)}=\frac{I}{2} .
$$

Par suite, le théorème de la moyenne montte que

$$
\lim _{l=+0} \frac{\mathrm{I}}{s(t)} \int_{(\mathrm{I})} F\left(J^{\prime}\right) d s=\frac{\mathrm{I}}{2} F(\jmath-0) ; \quad \lim _{t=+0} \frac{1}{s(t)} \int_{(2)} F\left(J^{\prime}\right) d s=\frac{\mathrm{I}}{2} F(\jmath+0) .
$$

La valeur moyenne est donc bien $\frac{f(x+0)+f(x-0)}{2}$. A l'extrémité $x=+\mathbf{I}$ $(x=-1)$ de l'intervalle, on voit sans autre que si $f(\mathrm{I}-0)[f(-\mathrm{I}+0)]$ existe, la valeur moyenne de $F(z)$ au pòle $z=0(\tau=\pi)$ existe et lui est égale.

De même, lorsqu'au point intérieur $x, f(x)$ est dérivée de son intégrale indéfinie, $F(\Xi)$ est pour la valeur correspondante égale à la dérivée superficielle de son intégrale superficielle. Il en sera de même pour $x=+\mathrm{I}, x=-\mathrm{I}$ lorsque $f(+\mathrm{I}), f(-\mathrm{I})$ sont respectirement égales aux limites de

pour $b=0$.

$$
\frac{\mathrm{I}}{b} \int_{\mathrm{I}-\mathrm{h}}^{\mathrm{I}} f(x) d x, \quad \frac{\mathrm{I}}{b} \int_{-1}^{-\mathrm{I}+b} f(x) d x
$$

On peut donc conclure le théorème général:

Théorème XI. - Soit $\left[a_{k}\right]$ la suite des cuefficienls de Legendre

$$
a_{k}=\frac{2 k+\mathrm{I}}{2} \int_{-1}^{+1} f(x) P_{k}(x) d x
$$

d'une fonction $f(x)$ absolument intégrable dans l'intervalle $(-\mathrm{I},+\mathrm{I})$ et soit

$$
S_{n}(x)=a_{0} P_{0}(x)+\sum_{k=1}^{n} \frac{n(n-\mathrm{I}) \ldots(n-k+\mathrm{I})}{(n+2)(n+3) \cdots(n+k+\mathrm{I})} a_{k} P_{k}(x) \quad(-\mathrm{I} \leq x \leq \mathrm{I}):
$$

I. En tout point de continuite' de $f(x)$, la suite $S_{n}(x)$ converge vers $f(x)$. La convergence est uniforme dans tout intervalle entièrement intérieur à un intervalle de continuitité de $f(x)$.

2. En tout point de discontinuité de première espèce, $S_{n}(x)$ a pour limite

$$
\frac{f(x+0)+f(x-0)}{2}
$$

lorsque $x$ est interieur. Lorsque $x= \pm$ I la limite est respectivement $f(\mathrm{I}-\mathrm{o}), f\left(\mathrm{I}_{\mathrm{I}} \mathrm{o}\right)$.

3. $S_{n}(x)$ a pour limite la dérivée de l'intégrale définie de $f(x)$ en tout point intérieur où elle existe. $S_{n}(-\mathrm{I})\left[S_{n}(+\mathrm{I})\right]$ converge vers la derivée à droite (à gaucbe) au point $x=-\mathrm{I}(x=+\mathrm{I})$ de l'integrale indéfinie de $f(x)$, quand elle existe.

I4. La sommation des dérivées, - Pour étendre nos théorèmes sur l'approximation des dérivées, remarquons que lorsque $F(z)$ possède une différentielle d'ordre $n$ sur la 
sphère, $\frac{d^{n} f(x)}{d x^{n}}$ existe et inversement. Il peut y avoir exception aux extrémitís de l'intervalle $(-\mathrm{I},+\mathrm{I})$. Ces points ne sont des points réguliers pour la sommation de la dérivée d'ordre $n$ que lorsque les $n$ premières dérivées de $F(\mathrm{~J})$ s'annulent pour $\mathrm{s}=0$ ou $z=\pi$, car, dans ce cas seulement, $F(z)$ posside une différentielle d'ordre $n$ aux pôles.

On peat voir que, lorsque pour un point $x$ intérieur

$$
\lim _{b=0} \frac{f(x+b)-f(x-b)}{2}
$$

existe, $F(\jmath)$ possède une dérivée généralisće sur la sphère pour toute direction $\theta$. De même l'analogue de la dérivée seconde généralisíe est ici

$$
\lim _{b=0} \frac{f(x+b)+f(x-b)-2 \bar{f}(x)}{b^{2}},
$$

$\vec{f}(x)$ désignant la valeur moyenne au point $x$. Les théorèmes du Chapitre II nous donnent donc, sans autre, le

Théorème XII. - La suite dérivée

$$
\frac{d^{s} S_{n}(x)}{d x^{s}}=\sum_{k=1}^{n} \frac{n(n-1) \ldots(n-k+\mathrm{I})}{(n+2)(n+3) \cdots(n+k+\mathrm{I})} a_{k} \frac{d^{s} P_{k}(x)}{d x^{5}} \quad(-\mathrm{I} \leqslant x \leqslant \mathrm{I})
$$

converge pour $n=\infty$ :

1. Vers $\frac{d^{s} f(x)}{d x^{s}}$ en tout point intérieur ou cette dérivée existe. La convergence est uniforme dans tout intervalle entièrement intérieur à un intervalle de continuité de $\frac{d^{s} f(x)}{d x^{s}}$.

2. Vers la dérivée généralisée d'ordre s de $f(x)$ en tout point intérieur où elle existe.

Quant au dernier point, nous n'en avons pas donné ici une démonstration complète. Le lecteur y suppléera sans difficulté. Pour la définition des dérivées généralisées d'ordre quelconque on pourra consulter le mémoire cité de M. de la Valtée Poussin.

Je n'insiste pas sur les conséquences que l'on peut tirer de ces théorèmes et sur leurs relations étroites avec les théorémes de Weierstrass et de Painlevé sur l'approximation des fonctions et de leurs dérivées.

Genève, le 26 juin rgrı.

MicheL PLANCHEREL. 